\title{
Percutaneous Closure of an Aorta Puncture Caused by a Central Venous
}

Maria Teresa Barrio-Lopez ${ }^{1 *}$, Jose Calabuig ${ }^{1}$, Gorka Bastarrika ${ }^{2}$, Miguel Artaiz-Urdazi ${ }^{1}$, Alberto Esteban-Fernandez ${ }^{1}$ and Gaudencio Espinosa ${ }^{3}$

${ }^{1}$ Department of Cardiology and Cardiac Surgery, Clinic University of Navarra, Avenida Pio XII 36, 31008, Pamplona, Spain

${ }^{2}$ Department of Radiology, Clinic University of Navarra, Avenida Pio XII 36, 31008, Pamplona, Spain

${ }^{3}$ Department of Vascular Surgery, Clinic University of Navarra, Avenida Pio XII 36, 31008, Pamplona, Spain

*Corresponding author: Maria Teresa Barrio Lopez, Department of Cardiology and Cardiac Surgery, Clinic University of Navarra, Avenida Pio XII 36, 31008, Pamplona, Spain, Tel: 34948255400 (Ext: 4382); Fax: 34948296 500; E-mail: terebarriol@gmail.com

Rec date: Jun 15, 2015; Acc date: Aug 18, 2015; Pub date: Aug 20, 2015

Copyright: (c) 2015 Barrio-Lopez MT, et al. This is an open-access article distributed under the terms of the Creative Commons Attribution License, which permits unrestricted use, distribution, and reproduction in any medium, provided the original author and source are credited.

\begin{abstract}
Severe complications associated with a central venous catheter implantation could be repaired successfully. We reported the case of an iatrogenic aortic arch puncture due to a central venous catheter from the left internal jugular vein. Because the patient had a poor prognosis a percutaneous procedure was performed. Aortography was done with a pigtail catheter for guidance. A wire was introduced through the venous catheter to the aorta and the catheter was removed. The hole in the aorta was closed with a Perclose closure system. The patient remained stable and the procedure was performed without complications.
\end{abstract}

Keywords: Aortic arch; Foreign body (aorta); Endovascular procedures

\section{Introduction}

Central venous catheterization provides a route for the administration of caustic and medications as well as allowing measurement of central venous pressure. It is estimated that millions of central venous catheters are inserted yearly in our hospitals [1]. These techniques are usually used in unstable patients so reduce or avoid complications associated with central venous catheters are imperative. We report a case of a central venous catheter which traversed the left internal jugular vein and entered the aortic arch, as well as the replacement of the central venous catheter and the percutaneus closure of the aortic arch.

\section{Case Report}

A 64-year-old woman with metastatic cervical cancer was admitted to the coronary care unit with the diagnosis of sepsis. The patient needed a central venous catheter for potassium replacement and central venous pressure monitoring. Patient had malnutrition and cachexia. In addition, platelet count was 58.000 per micro litre of blood. The attending physician decided to insert a right jugular central venous catheter. After several failed attempts he decided to try on the left side. Lateral to the carotid pulse, a 18-gauge needle was inserted. The returned blood was dark and without pulse. A J-shaped guide was introduced and then the needle was removed. After that, the tract was dilated with a 7F sheath/introducer combination. Once the tract was dilated, the dilator was removed and the catheter was threaded over the wire into the vessel. No mechanical complications occurred during this procedure. Central venous pressure was so high, and an ectopic intra-arterial insertion of the catheter was suspected. A plain chest $\mathrm{x}$ ray confirmed that catheter tip was near the aortic arch. Patient was stable and without signs of bleeding. To determine the exact localization of the catheter an angio-CT was performed. Figures 1 and 2 show as the catheter entered through the aortic arch and the tip was placed in descendant aorta. In the neck the catheter passed through the left internal jugular vein and it lacerated carotid artery.

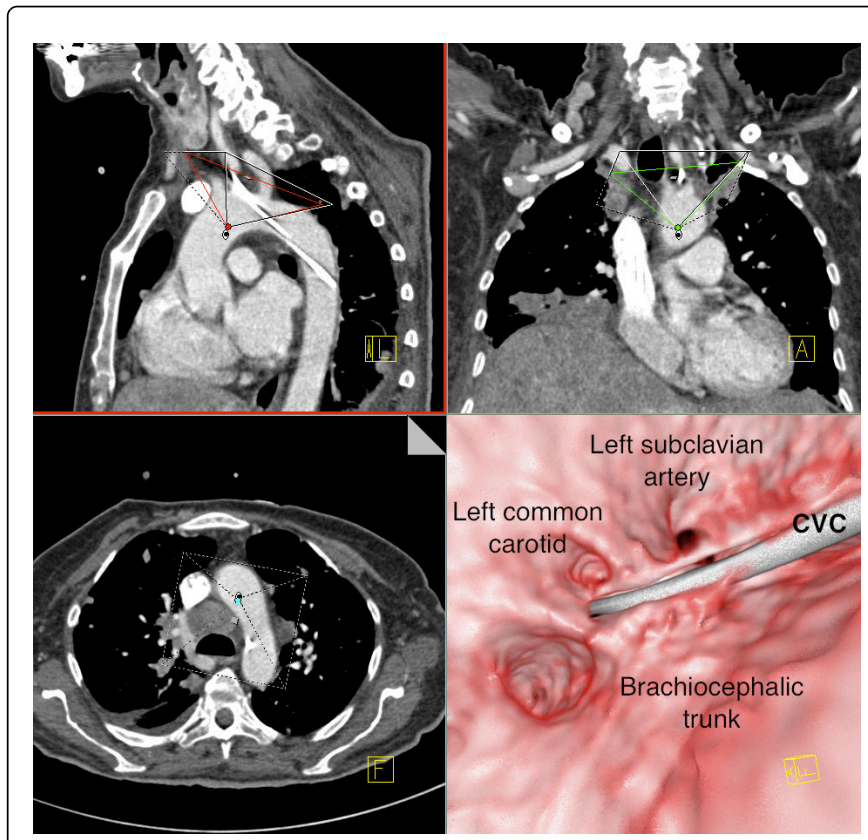

Figure 1: The catheter entered through the aortic arch and the tip was placed in descendant aorta.

The case was discussed with interventional radiologist, vascular surgeon and interventional cardiologist. Because patient had a terminal cancer and a debilitated state we concluded that this patient was a poor candidate for a major thoracic surgery. She was hemodinamically stable so we had to remove the catheter without cause more damage. First, an aortography was performed. It confirmed that the catheter tip was inside the aorta, near the origin of the carotid artery. The aortic access site was on the top of the aortic 
arch and the carotid artery was not perforated. A pigtail catheter was introduced through the left femoral artery and was advanced to the level of the aortic roof like a guidance.

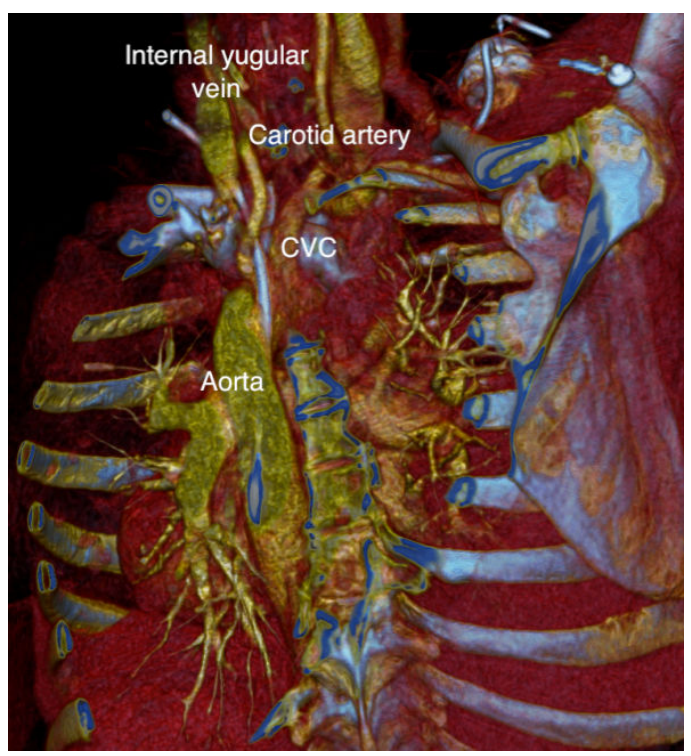

Figure 2: The catheter passed through the left internal jugular vein and it lacerated carotid artery.

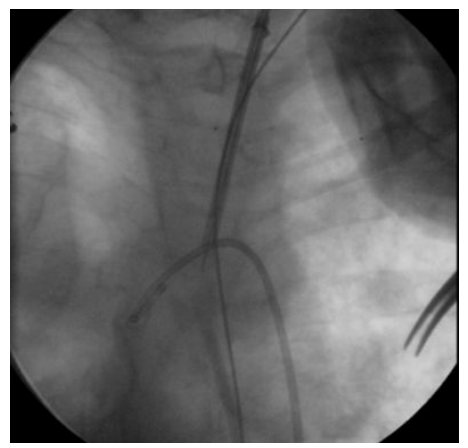

Figure 3: Without removing the wire the needles were deployed and then suture was tightened for closing the aorta.

\section{Discussion}

Aortic perforation during the placement of a jugular central venous catheter is a rare complication of this technique. Several cases of aortic perforation due a subclavian central venous catheter have been reported [2-4].

The anatomical proximity of the aorta and supraclavicular fossa could explain the complication. In these cases the accidental punctures were localized in the lateral wall of the ascending aorta and the bleeding from the perforation of the intrapericardial ascending aorta resulted in cardiac tamponade. In our case the perforation of the aorta was localized in the aortic arch with no bleeding. If bleeeding occurred an aortic or mediastinic haematoma could form. In addition, in cases of tamponade due to errant line placement, the physiological symptoms of tamponade started in the first fifteen minutes. Our patient was asymptomatic and without signs and symptoms of bleeding or tamponade. The chest X-ray and the angio-CT confirmed that the tip of the catheter was placed inside the descendant aorta. For that, we decided not to remove the catheter until we had a solution to close the aortic hole.

Then a J-shaped wire was introduced through the venous catheter to the aorta. After that we removed the catheter and kept the wire inside. To close the aortic puncture site we use Perclose ${ }^{\odot}$ suturemediated closure system. Under radiological guidance, we advanced the Perclose ProGlide sheath over the guide wire to the aortic arch. Without removing the wire we deployed the needles and then we tightened the suture for closing the aorta (Figure 3). After that, we waited about ten minutes and checked blood flow was absent. Then we removed the wire and an aortography confirmed the absence of contrast extravasation (Figure 4). At the site of skin insertion a venous bleeding stopped with compression. She remained hemodynamically stable and asymptomatic.

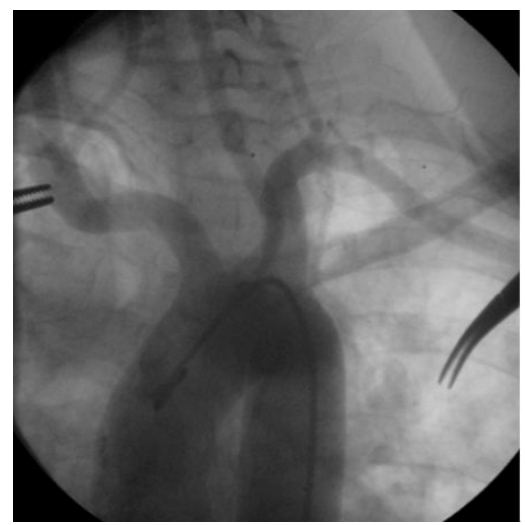

Figure 4: An aortography confirmed the absence of contrast extravasation after removing the wire.

As far as we know, only four case of aortic perforation due to a jugular central venous catheterization have been described [5-8]. In some cases an anatomic malformation could explain the aortic injury: thoracoabdominal aortic aneurysm [5], complete transposition of the great vessels [6]. In all cases repeatedly unsuccessful attempts was described [7].

Regarding to treatment, in the other cases of aortic damaged reported, different treatments were described. In some cases the tamponade caused an hemodynamically instability that required urgent sternotomy [4] and in others the patient died [7]. In other cases such as ours, patient was a poor candidate for a thoracic surgery due to a debilitated disease and a percutaneous closure was elected $[2,8]$. In the case described by Walser et al. a 7F balloon occlusion catheter was inserted in the hole of the aorta showing good result [2]. In the case reported by Patel et al. a closure device (Angio-SealTM, St Jude Medical) was performed successfully and without complication [8].

We chose Percolse device due to our extensive experience. Perclose device allows introduce the needles through the sheath $8 \mathrm{~cm}$ inside the vessel. For that we measured the distance between the skin access and the aortic puncture site in the angio-CT and we ensured that the needle reached the aortic arch. In addition, this system allows us closure the hole without remove the wire. If the procedure is 
Citation: Barrio-Lopez MT, Calabuig J, Bastarrika G, Artaiz-Urdazi M, Esteban-Fernandez A, et al. (2015) Percutaneous Closure of an Aorta Puncture Caused by a Central Venous . J Vasc Med Surg 3: 215. doi:10.4172/2329-6925.1000215

Page 3 of 3

unsuccessfully we could maintain the wire for new attempts and controlling massive bleeding or introduce a new venous catheter.

We recommended in patients with difficult accesses the use of ultrasonic locating devices [1] to avoid mechanical complications. Arterial puncture can occur during needle passage or during tract dilation if the dilator is advanced beyond the wire tip. In an arterial puncture is observed during attempted venous cannulation, the patient should be examined to detect early symptoms of cardiac tamponade (tachycardia, increased jugular venous pressure, hypontension, dyspnoea or presence of pulsus paradoxus). When inadvertent placement of the catheter in the arterial system is suspected because of chest X-ray findings or the character of blood return or anomalous central vein pressures, the catheter should not be removed, but its location precisely established by chest-computed tomography or by angiography. Open surgical removal may not be necessary if the patient is stable and vascular anatomy is favorable, because percutaneous extraction procedures can be safe and effective.

\section{References}

1. McGee DC, Gould MK (2003) Preventing complications of central venous catheterization. N Engl J Med 348: 1123-1133.
2. Walser EM, Crow WN, Zwischenberger JB, Conti VR (1996) Percutaneous tamponade of inadvertent transthoracic catheterization of the aorta. Ann Thorac Surg 62: 895-896.

3. Haaverstad R, Latto PN, Vitale N (2007) Right subclavian catheter perforation of the aorta due to an incorrect external landmark-guided insertion technique. CJEM 9: 43-55.

4. Seleem MI, Al-Hashemy AM, Al-Naemi A (2004) Ectopic intra-aortic insertion of a subclavian central venous catheter: Case report and review of the literature. J Thorac Cardiovasc Surg 127: 1515-1516

5. Iserson KV, Copeland J (1984) Pulmonary and aortic puncturesComplications of an attempt at internal jugular venipuncture. The Journal of Emergency Medicine 1: 227-231.

6. Schwartz AJ (1977) Percutaneous aortic catheterization - a hazard of supraclavicular internal jugular vein catheterization. Anesthesiology 46-77.

7. McDaniel MM, Grossman M (1978) Aortic dissection complicating percutaneous jugular vein catheterization. Anesthesiology 49: 213-214.

8. Patel SJ, Venn GE, Redwood SR (2003) Percutaneous Closure of an Iatrogenic Puncture of the Aortic Arch. Cardiovasc Intervent Radiol 26: 407-409. 